\title{
References:
}

[1] Kyeiborg M, Albrechtsen R, Couchman J, et al., Cellular roles of ADAM12 in health and disease, Int J Biochem Cell Biol, 2008

[2] Masahiro $\mathrm{H}$, Keiichiro $\mathrm{N}$, Joe $\mathrm{H}$, et al., Involvement of ADAM12 in Chondrocyte Differentiation by Regulation of TGF-beta1-Induced IGF-1 and RUNX-2 Expressions, Calcif Tissue Int, 2019

Fig. 1: Immunohistological staining of ADAM12 for synovial tissues

RA
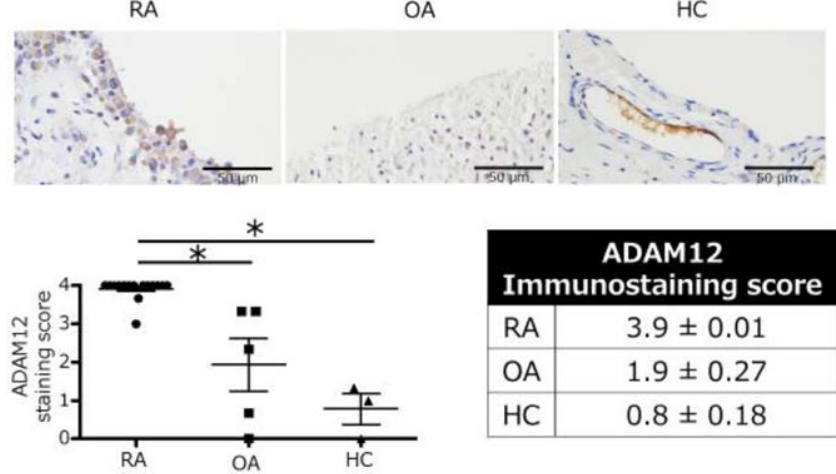

${ }^{*} \mathrm{p}<0.05$

(one way ANOVA post hoc Tukey test)

Fig. 2: ADAM12 relative mRNA expression and the proliferation of RASF after SiADAM12 transfection
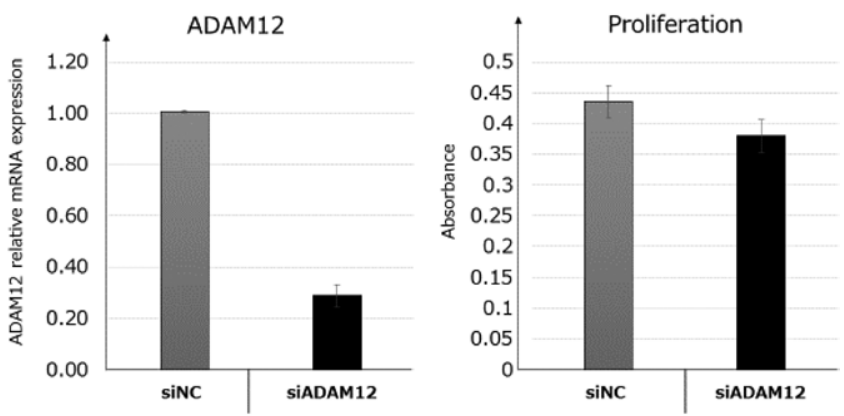

Disclosure of Interests: Masahito Watanabe: None declared, Keiichiro Nishida Grant/research support from: K. Nishida has received scholarship donation from CHUGAI PHARMACEUTICAL Co., Eisai Co., Mitsubishi Tanabe Pharma and AbbVie GK., Speakers bureau: K. Nishida has received speaking fees from CHUGAI PHARMACEUTICAL Co., Eli Lilly, Janssen Pharmaceutical K.K., Eisai Co. and AYUMI Pharmaceutical Corporation., Yoshihisa Nasu: None declared, Ryuichi Nakahara: None declared, Minami Matsuhashi: None declared, Yoshifumi Hotta: None declared, Toshifumi Ozaki: None declared DOI: 10.1136/annrheumdis-2020-eular.1968

\section{SAT0024 JAK-INHIBITION WITH BARICTINIB INHIBITS ACTIVATION OF NLRP1/CASPASE1/GDMSD PYROPTOSIS PATHWAY IN RHEUMATOID ARTHRITIS SYNOVIAL FIBROBLASTS}

W. Xie ${ }^{1}$, Z. Zhang ${ }^{1} .{ }^{1}$ Peking University First Hospital, Department of Rheumatology and Clinical Immunology, Beijing, China

Background: Synovial fibroblasts (SFs) play a major role in the pathogenesis of rheumatoid arthritis (RA) and develop an aggressive phenotype destroying cartilage and bone, thus termed RASFs.

Objectives: We aim to examine the presence of GSDMD-mediated pyroptosis and its role in activated RASFs.

Methods: Fibroblasts were isolated from RA synovium obtained from knee replacement surgeries. NLRP1, Caspase-1, GSDMD expression in synovial tissue and TNF-treated RASFs were assessed by qPCR and Western blot. Interleukin (IL)-1 was measured by ELISA in supernatant after pretreated with TNF and barictinib. LDH release was measured using the CytoTox 96 Non-Radioactive
Cytotoxicity Assay Kit. Endogenous NLRP1, Caspase-1, and GSDMD was knocked down using small interfering RNA.

Results: The expressions of NLRP1, pro-Caspase-1, Caspase-1 p10, GSDMD and its pyroptosis-inducing fragment GSDMD-N were greater in RA synovium than OA synovium. TNF-induced NLRP1, pro-Caspase-1, Caspase-1 p10, GSDMD, and GSDMD-N expression at the transcript and protein level in a time-dependent manner $(P<0.05)$. Meanwhile, the release of LDH and IL-1 were significantly increased in RASFs after treated with TNF. We also confirmed the presence of pyroptosis in electron microscopy. Furthermore, blocking the JAK pathway with barictinib significantly reduced TNF-induced pyroptosis at the transcriptional, protein and activity levels ( $P$ $<0.05)$. Finally, blocking the JAK pathway, we observed a reduction of IL-1 bioactivity in RASFs $(P<0.05)$.

Conclusion: Our results demonstrate an important role of GSDMD-mediated pyroptosis and shed lights on a potential pyroptosis-targeted treatment. Meanwhile, JAK inhibition alleviates inflammasome-induced pyroptosis by blocking pyroptosis pathway in RASFs.

References:

[1] Masters, S. L. et al. NLRP1 inflammasome activation induces pyroptosis of hematopoietic progenitor cells. Immunity,2012,37:1009-1023.

Disclosure of Interests: None declared

DOI: 10.1136/annrheumdis-2020-eular.5062 \section{ARTHRITIS THROUGH ACTIVATION OF ILC2}

\section{Y. Zhang ${ }^{1}$, Y. Qin ${ }^{1}$, Z. Chen ${ }^{1} .{ }^{1}$ The First Affiliated Hospital of USTC,} Rheumatology and Immunology, Hefei, China

Background: Reduction and dysregulation of ILC2 was linked to delayed resolution of arthritis. The neuropeptide Neuromedin $U$ (NMU) has been reported to rapidly activate ILC2 and initiate a Th2 type immune response through NMUR1 expressed on the surface of ILC2. However, one previous study reported that NMU promoted autoantibody-mediated arthritis.

Objectives: The aim of this work was to investigate the effect of NMU on collagen-induced arthritis (CIA) mice and the potential mechanisms.

Methods: $\mathrm{ClA}$ was induced in C57BL/6 WT and C57BL/6 ${ }^{\mathrm{Nmu}}$ deficient mice on day 1. WT mice were treated i.p. daily by NMU-23 (20ug/mice) or by PBS for 10 days from day 1 to 5 and day 21 to 25 . The clinical scores of CIA mice were assessed every two days from day 22 and determined on a scale of $0-4$ for each paw. The proportion of ILC2 as well as Th1, Th2, Th17 and Treg in spleen, mesenteric lymph node $(\mathrm{mLN})$ and joints of arthritic mice were analyzed by flow cytometry on day 42.

Results: NMU-23 dramatically inhibited clinical onset and severity of arthritis in treated WT mice compared with control mice. Interestingly, NMU-deficient mice also developed significantly less severe arthritis compared with WT control (Fig 1). Flow cytometry analyses showed that the proportion of ILC2, which defined as Lin-CD45+CD127+KLRG1+ICOS+ST2+, was elevated in the joint but not in the spleen and $\mathrm{mLN}$ of arthritic mice treated with NMU 23. In contrast, the proportion of ILC2 was significantly lower in the spleen of NMU-deficient mice than WT control. The percentage of Th2 cells in the spleen and $\mathrm{mLN}$ tend to be higher in NMU-23 treated mice, but there is no statistical significance. Surprisingly, Th1 cells were increased in the $\mathrm{mLN}$ of NMU-23 treated and NMU-deficient mice compared with control whereas Th17 was comparable among groups. In addition, the proportion of Treg was decreased in the joint of NMU-23 treated and NMU-deficient mice compared with control mice.

Conclusion: Our preliminary results show that repeated injection of NMU-23 during induction (early) and development (late) stage of CIA strongly suppressed clinical onset and severity of arthritis, which might be ascribed to activation of ILC2 in the joint. Further study is needed to explore other cellular and molecular mechanisms in the effect. References:

[1] Cardoso V, Chesne J, Ribeiro H et al (2017) Neuronal regulation of type 2 innate lymphoid cells via neuromedin U. Nature 549 (7671):277-281.

[2] Klose CSN, Mahlakoiv T, Moeller JB et al (2017) The neuropeptide neuromedin $U$ stimulates innate lymphoid cells and type 2 inflammation. Nature 549 (7671):282-286.

[3] Wallrapp A, Riesenfeld SJ, Burkett PR et al (2017) The neuropeptide NMU amplifies ILC2-driven allergic lung inflammation. Nature 549 (7672):351-356

[4] Sindhuja M Rao, Jennifer L Auger, Philippe Gaillard et al (2012) The Neuropeptide Neuromedin U Promotes Autoantibody-Mediated Arthritis. Arthritis Res Ther, 14 (1), R29. 